\title{
MULTI-ELEMENT COMPOSITION OF RED AND WHITE WINES FROM BUJORU, SMULTI AND OANCEA WINE CENTER, ROMANIA
}

\section{ALINA DONICI ${ }^{a}$, EMESE GAL ${ }^{b}$, CLAUDIA CIMPOIU ${ }^{b}$, CLAUDIU IOAN BUNEA $^{c, d}$, FLORIN DUMITRU BORA ${ }^{a},{ }^{*}$}

\begin{abstract}
The ICP-MS technique was used to determine elemental composition ( $\mathrm{Pb}, \mathrm{Sr}, \mathrm{Cd}, \mathrm{Ni}, \mathrm{Co}, \mathrm{Cu}, \mathrm{Ni}, \mathrm{Hg}, \mathrm{As}, \mathrm{Cr}$ and $\mathrm{Mn}$ ) of wines (Muscat Ottonel, Fetească Albă, Fetească Regală, Fetească Neagră, Merlot and Cabernet Sauvignon) wines produced in 2015, 2016 and 2017 from Bujoru, Smulti and Oancea wine-growing centers from Dealu Bujorului Vineyard. For all tested wine samples, the toxic metals contents were found in quantities below the limits established by legislation. The average data shows that the red wines contain highest concentration of $\mathrm{Cd}(0.17 \mu \mathrm{g} / \mathrm{L})$, while the content of $\mathrm{Cd}$ in white wines are $0.11 \mu \mathrm{g} / \mathrm{L}$. The concentration of $U$ was $0.25 \mu \mathrm{g} / \mathrm{L}$ in red wines and $0.24 \mu \mathrm{g} / \mathrm{L}$ in white wines, while the concentration of $\mathrm{Hg}$ was 0.24 $\mu \mathrm{g} / \mathrm{L}$ in red wines and $0.20 \mu \mathrm{g} / \mathrm{L}$ in white wines. The mean contents of $\mathrm{Ni}, \mathrm{Cr}$ and $\mathrm{Mn}$ were $312.32 \mu \mathrm{g} / \mathrm{L}, 526.19 \pm 2.63 \mu \mathrm{g} / \mathrm{L}$ and $0.59 \pm 0.08 \mathrm{mg} / \mathrm{L}$, respectively. The concentration for $\mathrm{Cu}$ ranged from $0.45 \pm 0.10 \mathrm{mg} / \mathrm{L}$ to $0.90 \pm 0.04 \mathrm{mg} / \mathrm{L}$, the last value being close to the law limit $(1 \mathrm{mg} / \mathrm{L})$. The four samples [Merlot $0.82 \pm 0.07 \mathrm{mg} / \mathrm{L}$ Bujoru wine-growing center (2015), Feteasca Alba 0.83 \pm 0.05 $\mathrm{mg} / \mathrm{L}$ Smulti wine-growing center (2015), Merlot 0.83 $\pm 0.05 \mathrm{mg} / \mathrm{L}$ Smulti winegrowing center (2015) and Cabernet Sauvignon $0.83 \pm 0.08 \mathrm{mg} / \mathrm{L}$ Oancea winegrowing center (2015)] showed relatively high concentration of $\mathrm{Cu}$. Reporting the obtained results [Cd average $0.13 \pm 0.02 \mu \mathrm{g} / \mathrm{L}(0.1 \mathrm{mg} / \mathrm{kg}$ M.L.A. $=$ Maximum Limit Allowed) Pb average 40.64 $\pm 1.85 \mu \mathrm{g} / \mathrm{L}$ (0.15 mg/kg M.A.L); As average $11.87 \pm 1.37 \mu \mathrm{g} / \mathrm{L}(0.2 \mathrm{mg} / \mathrm{kg}$ M.A.L); Cu average $0.67 \pm 0.09 \mathrm{mg} / \mathrm{L}(1 \mathrm{mg} / \mathrm{kg}$ M.A.L) to national and international legislation we can say that the wine from

a Research Station for Viticulture and Enology Targu Bujor, 65 G-ral Eremia Grigorescu Street, RO-805200, Galați Country Romania.

b Babeş-Bolyai University, Faculty of Chemistry and Chemical Engineering, 11 Arany Janos Street, RO-400028, Cluj-Napoca, Romania.

c University of Agricultural Sciences and Veterinary Medicine, Department of Horticulture and Landscaping, 3-5 Mănăștur Street, RO-400028, Cluj-Napoca, Romania.

d Institute of Advanced Horticultural Research of Transylvania, 3-5 Mănăștur Street, RO-400028,

* Corresponding author: boraflorindumitru@gmail.com
\end{abstract} Cluj-Napoca, Romania. 


\begin{abstract}
Dealu Bujorului vineyard falls within the limits set by the law. The content of potentially toxic elements such as $\mathrm{Cd}, \mathrm{Pb}, \mathrm{U}, \mathrm{Hg}, \mathrm{As}, \mathrm{Cu}, \mathrm{Ni}, \mathrm{Cr}$ and $\mathrm{Mn}$ are lower than values found in literature, highlighting the safety and quality of the analysed Romanian wines.
\end{abstract}

Keywords: Dealu Bujorului vineyard, elemental composition, geographical traceability, wine.

\title{
INTRODUCTION
}

The origin and authenticity of food products are topics of great interest in the food industry, not only for consumers but also for producers and distributors. Additionally, the use of geographical designations allows producers to obtain market recognition and often a premium price [1]. Wine is a product widely consumed around the world and has been extensively investigated because of frauds, including adulteration, false declaration of geographical origin and false age declaration. The huge diversity of production areas poses a challenge in establishing the provenance of wine as their grape variety, soil and climate conditions, history, yeast, oenological practices, storage and transport [2,3]. To take marketing advantage of the recent large improvements in wine quality in several "New World" wine regions, many local producers have changed to declare the specific region of origin rather than just naming the grape variety [4]. The denomination origin controlled (DOC) system are applied in many countries to control and ultimately guarantee the quality, origin and also to prevent de fraud. Chemical characterization is one of the requirements to obtain DOC certification of wines. Nowadays, there is a wide range of combined techniques to identify wine authenticity [5].

The inorganic chemical pattern of a wine is a reflection of the local geochemistry of the soil, climate and winemaking process. The elemental composition is mainly influenced by the bioavailability of inorganic compounds of the soil and the demands of the vine [6]. The initial concentration of elements can be modified during the winemaking process by the addition of bentonite or similar compounds, used to clarify the wine or by coprecipitation present in the must [7]. Not all elements are metabolized or modified during the winemaking process [8]. In wine from three southern Italian wine-producing regions were identified $\mathrm{Li}$ and $\mathrm{Rb}$ as elements facilitating a successful classification [9] wine from Spain were able to discriminate from two different Valencia by using $\mathrm{Li}$ and $\mathrm{Mg}$ content. However, factors such as environmental pollution, agricultural practices, climatic changes and winemaking process may change the multi-element 
composition of wines. Nevertheless, significant correlations were obtained between the elemental composition of vineyard soil and wine [10].

The presence of lead in wine is associated with two major sources as follows: natural sources, which are due to the weathering of rocks, and human activity, which results from the use of fertilizers, pesticides and agricultural and food additives and environmental pollution [11].

The goal of this study is to determine the elemental composition ( $\mathrm{Cd}, \mathrm{Pb}, \mathrm{U}, \mathrm{Hg}, \mathrm{As}, \mathrm{Sr}, \mathrm{Co}, \mathrm{Cu}, \mathrm{Ni}$, Ce and $\mathrm{Mn}$ ) of wine from Bujoru, Smulti and Oancea wine-growing centers from Dealu Bujorului vineyardand to assess their ability to discriminate between geographical origin of wines. The wines from Smulti and Oancea wine-growing centers have not been analyzed yet regarding concentration of the elemental composition.

\section{RESULTS AND DISCUSSION}

\section{Heavy metals content in wine samples from Bujoru, Smulti and Oancea Wine Center}

Table 1 and 2 summarize the total contents of $\mathrm{Cd}, \mathrm{U}, \mathrm{Hg}$, As, Co, $\mathrm{Cu}, \mathrm{Ni}, \mathrm{Cr}$ and $\mathrm{Mn}$ in wine samples. Generally, the heavy metals are found in wine due to their presence in grapes, from where they are extracted during maceration [12]. These elements are found in grapes as a result of their accumulation in the plant from the soil, or they could be absorbed from the agents used in protecting the vine from diseases. In the course of the maceration, extracted elements are absorbed in the cell membrane of yeast, and in a while, their declines as a consequence of precipitation in conjunction with the yeast cell. The contact of wine with equipment, the addition of fining agents or the changing of filters during post fermentation processes could increase the content of some elements [12].

The mean contents of $\mathrm{Cd}, \mathrm{U}$ and $\mathrm{Hg}$ were $0.13 \pm 0.02 \mu \mathrm{g} / \mathrm{L}, 0.24 \pm 0.03$ $\mu \mathrm{g} / \mathrm{L}$ and $0.20 \pm 0.03 \mu \mathrm{g} / \mathrm{L}$. The highest concentrations of $\mathrm{Cd}$ and $U$ were recorded in the wines obtained in 2016 from Smulti wine-growing center, namely Feteasca Neagra $(0.22 \pm 0.04 \mu \mathrm{g} \mathrm{Cd} / \mathrm{L}$ and $0.41 \pm 0.06 \mu \mathrm{g} \mathrm{U} / \mathrm{L}$ and Merlot $(0.21 \pm 0.02 \mu \mathrm{g} \mathrm{Cd} / \mathrm{L}$ and $0.45 \pm 0.05 \mu \mathrm{g} \mathrm{U} / \mathrm{L})$, while the highest concentrations of $\mathrm{Hg}$ were recorded in wines obtained from Bujoru winegrowing center (Feteasca Alba - $0.43 \pm 0.05 \mu \mathrm{g} \mathrm{Hg} / \mathrm{L}$ ) in both years 2015 and 2016. The average data shows that the highest concentration of $\mathrm{Cd}$ was obtained to red wines $(0.17 \mu \mathrm{g} / \mathrm{L})$ compared to white wines white $(0.11 \mu \mathrm{g} / \mathrm{L})$, the highest concentration of $U$ was obtained to red wines $(0.25 \mu \mathrm{g} / \mathrm{L})$ compared to white wines white $(0.24 \mu \mathrm{g} / \mathrm{L})$ while the highest concentration of $\mathrm{Hg}$ was obtained to white wines $(0.24 \mu \mathrm{g} / \mathrm{L})$, compared to white wines white $(0.20 \mu \mathrm{g} / \mathrm{L})$. The results agree with some studies [12] $0.25 \mu \mathrm{g} / \mathrm{L}(\mathrm{Cd}), 0.11 \mu \mathrm{g} / \mathrm{L}$ $(U)$, [13] $0.14 \mu \mathrm{g} / \mathrm{L}(\mathrm{Cd})$, but compared with other result [14], the concentration 
of Cd was significantly higher than ours $10.60 \mu \mathrm{g} / \mathrm{L}(\mathrm{Cd})$. Regarding $\mathrm{Hg}$ concentration the results agree with those made in Romania [15] for Sauvignon Blanc $0.56 \mu \mathrm{g} / \mathrm{L}$, Feteasca Alba $0.22 \mu \mathrm{g} / \mathrm{L}$ and Riesling $0.16 \mu \mathrm{g} / \mathrm{L}$.

Table 1. Variation of the metal content of white and red wines from Dealu Bujorului Vineyard $(\mu \mathrm{g} / \mathrm{L})$

\begin{tabular}{|c|c|c|c|c|c|c|}
\hline$\stackrel{\mathbb{\varpi}}{\stackrel{\infty}{<}}$ & $\frac{\sqrt{0}}{\frac{0}{\pi}}$ & $\begin{array}{l}\stackrel{\bar{\varpi}}{\circlearrowright} \\
\succ\end{array}$ & $\begin{array}{c}\mathrm{Cd} \\
\text { M.L.A. } \\
\mathbf{0 . 0 1} \mathrm{mg} / \mathrm{L}\end{array}$ & $\begin{array}{l}\text { U } \\
\text { M.L.A. } \\
\quad-\end{array}$ & $\begin{array}{c}\mathrm{Hg} \\
\text { M.L.A. } \\
-\end{array}$ & $\begin{array}{c}\text { As } \\
\text { M.L.A. } \\
0.2 \text { mg/L }\end{array}$ \\
\hline \multirow{18}{*}{ 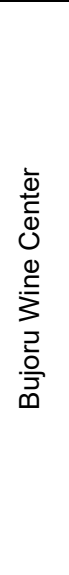 } & \multirow{4}{*}{$\begin{array}{c}\mathrm{M} \\
\mathrm{Ott} .\end{array}$} & 2015 & $0.11 \pm 0.02^{m} \eta$ & $0.18 \pm 0.03^{\mathrm{klmnop}} \delta \varepsilon \zeta$ & $0.34 \pm 0.08^{\text {bcd }} \beta$ & $14.16 \pm 2.26^{\text {efghijk }} \beta \gamma \delta$ \\
\hline & & 2016 & $0.12 \pm 0.01^{\mathrm{km}} \zeta \eta$ & $27 \pm 0.04^{\text {bcdefghij }} \alpha \beta y$ & $0.32 \pm 0.05^{\text {bcdeff }} \beta$ & $15.48 \pm 0.85^{\text {defghi }} \beta y$ \\
\hline & & 2017 & $0.12 \pm 0.02^{\mathrm{klm}} \zeta \mathrm{n}$ & $0.24 \pm 0.09^{\text {cdefighijkmn }} \beta \gamma \delta \bar{\delta} \varepsilon$ & $0.30 \pm 0.02^{\text {bcdefg }} \beta$ & $21.85 \pm 1.74^{\mathrm{a}} \alpha$ \\
\hline & & 2015 & $0.12 \pm 0.0^{\operatorname{lm}} \zeta \eta$ & $0.18 \pm 0.06^{\mathrm{klmnop}} \delta \varepsilon \zeta$ & $0.43 \pm 0.05^{\mathrm{a}} \alpha$ & $16.43 \pm 0.92^{\text {cdefg }} \beta$ \\
\hline & \multirow[t]{3}{*}{ FA. } & 2016 & $\operatorname{LOQ}^{n} \theta$ & $0.24 \pm 0.02^{\text {cdefghijkkmn }} \beta \gamma \delta \varepsilon$ & $0.43 \pm 0.04^{a} \alpha$ & $13.79 \pm 1.57^{\text {ghijk }} \gamma \delta \varepsilon \varepsilon$ \\
\hline & & 2017 & $0.13 \pm 0.0^{\mathrm{j} \mathrm{k} / \mathrm{m}} \delta \varepsilon \zeta \eta$ & $0.17 \pm 0.02^{1 \mathrm{mnop}} \varepsilon \zeta$ & $0.31 \pm 0.03^{\text {bcdef }} \beta$ & $13.36 \pm 1.15^{\mathrm{hijkl}} \gamma \delta \varepsilon$ \\
\hline & & 2015 & $\operatorname{LOQ}^{n} \theta$ & $0.21 \pm 0.05^{\text {ghjiklmnop }} \gamma \delta \varepsilon \zeta$ & $0.26 \pm 0.08^{\text {fghij }}$ & $8.77 \pm 0.65^{p} \theta$ \\
\hline & \multirow[t]{3}{*}{ F R. } & 2016 & $\operatorname{LOQ}^{n} \theta$ & $0.22 \pm 0.04^{\text {efghij }}$ & $0.43 \pm 0.04^{\mathrm{a}} \alpha$ & $13.79 \pm 1.57^{\text {ghijk }} \gamma \delta \varepsilon$ \\
\hline & & 2017 & $0.13 \pm 0.01^{1 \mathrm{jklm}} \varepsilon \zeta \eta$ & $0.35 \pm 0.02^{b} \alpha$ & $0.31 \pm 0.05^{\text {bcdefg }} \beta$ & $15.23 \pm 1.15^{\text {defghi }} \beta y$ \\
\hline & & 2015 & $0.16 \pm 0.01^{\text {defghijkl }}$ & $0.24 \pm 0.05^{\text {cdefghijkkmn }} \beta \gamma \delta \varepsilon$ & $0.33 \pm 0.04^{\text {bcde }} \beta$ & $14.37 \pm 2.09^{\text {efghijk }} \beta \gamma \delta \bar{\varepsilon}$ \\
\hline & \multirow[t]{3}{*}{ F N. } & 2016 & $0.15 \pm 0.02^{\text {defc }}$ & $0.26 \pm 0.03^{\mathrm{bcde}}$ & $0.27 \pm 0.08^{\text {cdefghi }} \beta$ & $10.03 \pm 0.47^{\mathrm{nop}} n \theta$ \\
\hline & & 2017 & 0. & $.03^{\mathrm{bcd}}$ & $0.05^{\text {bcdefg }} \beta$ & $50.87^{\text {nop }} n \theta$ \\
\hline & & 2015 & $0.16 \pm 0.02^{\text {cdefghijkl }} \gamma \delta$ & $0.17 \pm 0.01^{1 \mathrm{mnop}} \varepsilon \zeta$ & $\mathrm{LOQ}^{\mathrm{s}} \delta$ & $12.02 \pm 0.50^{\mathrm{klmno}} \delta \varepsilon \zeta$ \\
\hline & \multirow[t]{3}{*}{ M } & 2016 & $0.21 \pm 0.02^{\mathrm{ab}} \alpha$ & $0.16 \pm 0.01^{\text {nop }} \varepsilon \zeta$ & $0.30 \pm 0.04$ & $21.02 \pm 1.36^{\mathrm{ab}} \alpha$ \\
\hline & & 2017 & & $03^{\mathrm{mnop}} \varepsilon \zeta$ & $0.03^{\text {efghij }} \beta$ & \\
\hline & & 2015 & $0.13 \pm 0.02^{\mathrm{ijklm}} \varepsilon \zeta \eta$ & $0.30 \pm 0.08^{\text {bcdef }} \alpha \beta$ & $\mathrm{LOQ}^{\mathrm{s}} \delta$ & $10.45 \pm 0.81^{1 \mathrm{mnop}} \eta \theta$ \\
\hline & \multirow[t]{2}{*}{ C S. } & 2016 & $0.17 \pm 0.02^{\text {bcdefghij }} \beta \gamma$ & $0.27 \pm 0.02^{\text {bcdefghij }} \alpha \beta \gamma$ & $0.15 \pm 0.02^{\mathrm{opr}} \gamma$ & $9.90 \pm 1.22^{\text {nop }} \eta \theta$ \\
\hline & & 2017 & $.03^{\text {bcdefghij }} \beta y$ & $03^{\text {defghijklmno }} \beta \gamma \delta \varepsilon \zeta$ & LO & $0.61^{\mathrm{ijklmn}} \zeta n \theta$ \\
\hline \multirow{18}{*}{ 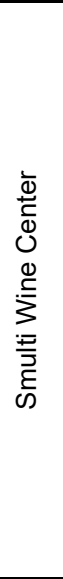 } & \multirow{4}{*}{$\begin{array}{c}\text { M } \\
\text { Ott. }\end{array}$} & 2015 & $22^{i j k l m} \gamma \delta \varepsilon$ & $08^{\text {bodefghijkl }} \alpha \beta \gamma \delta$ & $02^{\mathrm{pr}} n \theta$ & $1.57^{\text {hijkl } \delta \varepsilon \zeta \eta}$ \\
\hline & & 2016 & & $\alpha \beta \gamma \delta$ & 0. & $29^{\text {fghijk }} \delta \varepsilon$ \\
\hline & & 2017 & LOC & $0.04^{\mathrm{op}} \delta \varepsilon$ & 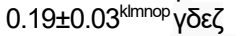 & $17.40 \pm 1.99^{\text {cd }} \beta \gamma$ \\
\hline & & 2015 & $0.0^{\mathrm{jklm}} \varepsilon$ & $.02^{\text {ghjiklmnop }} \beta \gamma \delta \varepsilon$ & $0.17 \pm 0.02^{\operatorname{lmnopr}} \delta \varepsilon \zeta \eta \theta$ & $.56^{p_{1}}$ \\
\hline & \multirow[t]{3}{*}{ F A. } & 2016 & $0.03^{\text {efghijklm }}$ & $0.02^{b c} \alpha$ & $0.16 \pm 0.01$ & $\pm 0.76^{\mathrm{mnop}} \eta \theta_{\mathrm{I}}$ \\
\hline & & 2017 & LC & $0.27 \pm 0.05^{\text {bcdefghij }} \alpha \beta \gamma$ & $0.22 \pm 0.04^{\mathrm{hijklm}} \beta \gamma \delta$ & $10.82 \pm 1.22^{\mathrm{Innop}} \varepsilon \zeta \eta{ }_{\mathrm{I}}$ \\
\hline & & 2015 & $0.16 \pm 0.02^{\text {bcdefghijk }}$ & $0.18 \pm 0.03^{\mathrm{klmnop}} \delta \varepsilon$ & $0.15 \pm 0.01^{\text {nopr }} \zeta n \theta$ & $0.30^{\mathrm{p}_{1}}$ \\
\hline & \multirow[t]{3}{*}{ F R. } & 2016 & $\mathrm{LOQ}^{n} \zeta$ & $.07^{\mathrm{bcd}}$ & $0.02^{\mathrm{k} k m n o p}$ yठ $\varepsilon \zeta$ & $69^{j \mathrm{klkm}} \delta \varepsilon \zeta \eta \cap \theta$ \\
\hline & & 2017 & & 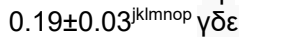 & $0.01^{r}$ & $\pm 0.92^{\operatorname{lmnop}} \eta \theta_{1}$ \\
\hline & & 2015 & $0.18 \pm 0.04^{\text {abcdefgh }} \alpha \beta \gamma \delta$ & $0.16 \pm 0.01^{\text {nop }} \delta \varepsilon$ & $0.20 \pm 0.03^{\mathrm{kkmnop}} \gamma \delta \varepsilon \zeta$ & $15.82 \pm 3.24^{\text {defgh }} \gamma \delta$ \\
\hline & \multirow[t]{3}{*}{ F N. } & 2016 & $0.17 \pm 0.05^{\text {abcdefgh }} \alpha \beta \gamma \delta \varepsilon$ & $.02^{p} \delta \varepsilon$ & $0.17 \pm 0.02^{\text {Imnopr }} \delta \varepsilon \zeta$ & $19.02 \pm 3.12^{\mathrm{bc}} \alpha \beta$ \\
\hline & & 2017 & & $02^{\text {ghijkln }}$ & $0.02^{\text {hijkl }} \beta \gamma \delta$ & $1.34^{\mathrm{ab}} \alpha$ \\
\hline & & 2015 & $0.18 \pm 0.04^{\mathrm{ab}}$ & ${ }^{i j k} \alpha \beta \gamma \delta$ & ${ }^{n} \beta \gamma \delta$ & $13.76 \pm 1.61^{\text {ghijk }} \delta \varepsilon \zeta$ \\
\hline & \multirow[t]{3}{*}{ M } & 2016 & $0.19 \pm 0.04^{\mathrm{abcd}} \alpha \beta$ & 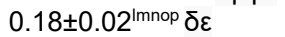 & $3^{\text {hijkl }} \beta \times \delta$ & $12.58 \pm 1.81^{\mathrm{jk} k \mathrm{kn}} \varepsilon \zeta \cap \theta$ \\
\hline & & 2017 & & ן & $\beta ү \delta$ & ${ }^{\circ} \varepsilon \zeta \cap \theta_{1}$ \\
\hline & & 2015 & $0.14 \pm 0.02^{\text {efghijklm }} \beta \gamma \delta \varepsilon$ & $0.21 \pm 0.06^{\text {ghij }}$ & $0.34 \pm 0.02^{\mathrm{bc}} \alpha$ & $10.23 \pm 1.70^{\text {nop }} \eta \theta$ \\
\hline & \multirow[t]{2}{*}{ C S. } & 2016 & $0.18 \pm 0.03^{\text {abcdefg }} \alpha \beta \gamma \delta \varepsilon$ & $.02^{\mathrm{hijk} k m n o p} \beta \gamma \delta \varepsilon$ & $0.06^{\text {bcdefg }} \alpha$ & $=0.30^{\text {nop }} \theta_{1}$ \\
\hline & & & & & & $\zeta \eta$ \\
\hline \multirow{6}{*}{ 产 } & \multirow{4}{*}{$\begin{array}{c}\mathrm{M} \\
\text { Ott. }\end{array}$} & 2015 & $\mathrm{jkm} n$ & $02^{\text {hijklmnop }}$ & $\mathrm{LC}$ & $12.66 \pm 0.46^{\mathrm{ijk} \mid m n} \beta \gamma \delta$ \\
\hline & & 2016 & $0.17 \pm 0.01^{\text {bodefghii }} \beta y \delta \varepsilon \zeta$ & $0.24 \pm 0.07^{\text {vdefghijklmn }} \delta \varepsilon \zeta$ & $0.20 \pm 0.05^{\mathrm{jklmnop}} \delta \varepsilon \zeta$ & $12.69 \pm 1.72^{\mathrm{ijklmn}} \beta \gamma \delta$ \\
\hline & & 2017 & $0.20 \pm 0.01^{\mathrm{abc}} \alpha \beta \gamma$ & $0.33 \pm 0.02^{b} y$ & $0.21 \pm 0.05$ & LOC \\
\hline & & 2015 & & $0.22 \pm 0.04^{\text {fghijklmnop }} \varepsilon \zeta$ & $\mathrm{LOQ}^{\mathrm{s}} \theta$ & $9.59 \pm 1.69^{o p} \varepsilon$ \\
\hline & \multirow[t]{2}{*}{ F A. } & 2016 & $0.17 \pm 0.01^{\text {bcdefghij }} \beta \gamma \delta \varepsilon$ & $0.20 \pm 0.02^{\mathrm{hijk} k \mathrm{mnop}} \zeta$ & $0.17 \pm 0.01^{1 \mathrm{mnopr}} \varepsilon \zeta$ & $13.21 \pm 1.45^{\mathrm{hijklm}} \beta \gamma$ \\
\hline & & 2017 & $0.13 \pm 0.02^{\mathrm{jklm}} \zeta \eta$ & $0.48 \pm 0.03^{\mathrm{a}} \alpha$ & $0.19 \pm 0.03^{\mathrm{jklmnop}} \delta \varepsilon \zeta$ & $10.36 \pm 0.76^{\mathrm{mnop}} \delta \varepsilon$ \\
\hline \multirow{3}{*}{ 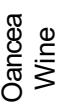 } & \multirow{3}{*}{ F R. } & 2015 & $0.16 \pm 0.01^{\text {bodefghijk }} \gamma \bar{c}$ & $0.30 \pm 0.02^{\mathrm{bcde}}$ & $0.35 \pm 0.02^{b} \alpha$ & $16.97 \pm 3.84^{\text {cde }} \alpha$ \\
\hline & & & & & & 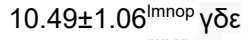 \\
\hline & & 2017 & $0.13 \pm 0.02^{\mathrm{jklm}} \zeta \eta$ & $0.29 \pm 0.02^{\text {bcdefg }} \gamma \delta \varepsilon$ & 0.02 & 10 \\
\hline
\end{tabular}




\begin{tabular}{|c|c|c|c|c|c|c|}
\hline \multirow[t]{2}{*}{ 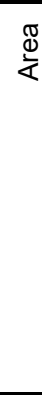 } & $\begin{array}{l}\frac{\overrightarrow{0}}{\frac{0}{\frac{1}{0}}} \\
>\end{array}$ & \} $&{\begin{array}{c}\text { Cd } \\
\text { M.L.A. } \\
0.01 \mathrm{mg} / \mathrm{L}\end{array}} &{\begin{array}{l}\text { U } \\
\text { M.L.A. } \\
-\end{array}} &{\begin{array}{l}\mathrm{Hg} \\
\text { M.L.A. } \\
\quad-\end{array}} &{\begin{array}{c}\text { As } \\
\text { M.L.A. } \\
0.2 \mathrm{mg} / \mathrm{L}\end{array}} \\
{\hline} &{\text { C S. }} &{\begin{array}{l}2015 \\
2016 \\
2017 \\
2015 \\
2016 \\
2017 \\
2015 \\
2016 \\
2017 \\
\end{array}} &{\begin{array}{l}0.14 \pm 0.03^{\text {fghijklm }} \varepsilon \zeta \eta \\
0.22 \pm 0.04^{\mathrm{a}} \alpha \\
0.20 \pm 0.02^{\mathrm{abcd}} \alpha \beta \gamma \\
0.19 \pm 0.03^{\mathrm{abcd}} \alpha \beta \gamma \delta \\
0.21 \pm 0.02^{\mathrm{ab}} \alpha \beta \\
0.19 \pm 0.02^{\mathrm{abcd}} \alpha \beta \gamma \delta \\
0.17 \pm 0.02^{\text {bcdefghij }} \gamma \delta \varepsilon \zeta \\
0.19 \pm 0.02^{\text {abcde }} \alpha \beta \gamma \delta \\
0.18 \pm 0.04^{\text {abcdef }} \alpha \beta \gamma \delta \varepsilon \\
\end{array}} &{\begin{array}{l}0.28 \pm 0.03^{\text {bcdefgh }} \gamma \delta \varepsilon \\
0.41 \pm 0.06^{\mathrm{a}} \beta \\
0.29 \pm 0.02^{\text {bcdefg }} \gamma \delta \varepsilon \\
0.45 \pm 0.05^{\mathrm{a}} \alpha \beta \\
0.26 \pm 0.05^{\text {bdeffohijk }} \beta \gamma \delta \varepsilon \zeta \\
0.30 \pm 0.04^{\text {bcdef }} \gamma \delta \\
0.32 \pm 0.03^{\text {bc }} \gamma \\
0.29 \pm 0.02^{\text {bcdefg }} \gamma \delta \varepsilon \\
0.32 \pm 0.07^{\text {bc }} \gamma\end{array}} &{\begin{array}{l}0.28 \pm 0.04^{\text {bcdefgh }} \beta \gamma \\
0.33 \pm 0.03^{\text {bcd }} \alpha \beta \\
0.36 \pm 0.04^{\text {b } \alpha} \\
0.15 \pm 0.03^{\text {mnopr }} \zeta \\
0.17 \pm 0.01^{1 \mathrm{mnopr}} \varepsilon \zeta \\
0.24 \pm 0.02^{\text {hijk }} \gamma \delta \\
0.20 \pm 0.03^{\text {jklmnop }} \delta \varepsilon \zeta \\
0.23 \pm 0.02^{\text {hijkl }} \gamma \delta \\
L O Q^{s} \theta\end{array}} &{\begin{array}{l}16.67 \pm 0.84^{\text {cdef }} \alpha \\
12.24 \pm 1.22^{\text {jkmno }} \beta \gamma \delta \varepsilon \\
16.92 \pm 2.51^{\text {cde }} \alpha \\
\text { LOQ' } \alpha \\
9.41 \pm 1.16^{\text {op }} \varepsilon \\
L^{\prime} Q^{\prime} \zeta \\
10.83 \pm 1.72^{\text {Imnop }} \gamma \delta \varepsilon \\
14.96 \pm 1.70^{\text {defghij }} \alpha \beta \\
L^{\prime O O Q} \zeta\end{array}} \\
{\hline \multicolumn{3}{|c|}{\begin{array}{c}\text { Average } \\
\text { Minimum Values } \\
\text { Maximum Values }\end{array}}\begin{array} { c } { \text { Average } } \\
{ \text { Minimum Values } } \\
{ \text { Maximum Values } } \end{array}} &{\begin{array}{l}\mathbf{0 . 1 3} \pm \mathbf{0 . 0 2} \\
0.11 \pm 0.02 \\
0.22 \pm 0.04\end{array}} &{\begin{array}{l}\mathbf{0 . 2 4} \pm \mathbf{0 . 0 3} \\
0.14 \pm 0.02 \\
0.48 \pm 0.03\end{array}} &{\begin{array}{l}\mathbf{0 . 2 0} \pm 0.03 \\
0.13 \pm 0.02 \\
0.48 \pm 0.03\end{array}} &{\begin{array}{c}11.87 \pm 1.37 \\
8.77 \pm 0.65 \\
21.85 \pm 1.74\end{array}} \\
{\hline \multicolumn{3}{|c|}{\begin{array}{c}\mathrm{F} \\
\text { Sig. }\end{array}}\begin{array} { c } { \mathrm { F } } \\
{ \text { Sig. } } \end{array}} &{\begin{array}{c}23.242 \\
\star \star \star *\end{array}} &{\begin{array}{c}9.324 \\
* \star \star\end{array}} &{\begin{array}{c}29.901 \\
* \star \star\end{array}} &{\begin{array}{c}33.878 \\
* * * \\
\end{array}} \\
{\hline \multicolumn{2}{|c|}{\text { Area }}\text { Area }} &{\begin{array}{c}\mathrm{F} \\
\text { Sig. }\end{array}} &{\underset{* \star *}{87.159}} &{\begin{array}{c}71.396 \\
* * \star\end{array}} &{\begin{array}{c}56.695 \\
* \star *\end{array}} &{\underset{* * *}{80.655}} \\
{\hline \multicolumn{2}{|c|}{\text { Variety }}\text { Variety }} &{\begin{array}{c}\mathrm{F} \\
\text { Sig. }\end{array}} &{\underset{* \star \star}{97.092}} &{\begin{array}{c}1.469 \\
\text { in }\end{array}} &{\begin{array}{c}34.672 \\
\star \star \star\end{array}} &{\begin{array}{c}62.417 \\
* * *\end{array}} \\
{\hline \multicolumn{2}{|c|}{\text { Year }}\text { Year }} &{\begin{array}{c}\mathrm{F} \\
\text { Sig. }\end{array}} &{\begin{array}{c}1.465 \\
\text { in }\end{array}} &{\begin{array}{c}2.954 \\
\text { in }\end{array}} &{\underset{* \star *}{35.014}} &{\begin{array}{c}32.436 \\
* * *\end{array}} \\
{\hline} &{\begin{array}{l}{[18]} \\
{[23]} \\
{[15]} \\
{[24]}\end{array}} &{ } &{\begin{array}{l}0.41 \\
0.22\end{array}} &{\begin{array}{c}0.86 \pm 0.55 \\
0.55\end{array}} &{0.56} &{\begin{array}{l}7.10 \\
5.24\end{array}} \\
$\hline
\end{tabular}

Average value \pm standard deviation $(n=3)$. Romans letters represent the significance of the variety difference $(p \leq 0.05)$. Greeks letters represent the significance of the same variety cultivated in other year's difference ( $p$ $\leq 0.05$ ). The difference between any two values, followed by at least one common letter, is insignificant. M.L.A. - maximum limit allowed (OIV, 2005); LOQ - lower than the limit of quantification; $\mathrm{Cu}^{*}=(\mathrm{mg} / \mathrm{L}) ; \mathrm{Cu}^{* *}=(\mu \mathrm{g} / \mathrm{L})$; $\mathrm{Mn}^{\star \star *}=(\mathrm{mg} / \mathrm{L}) . \mathrm{M}$ Ott. = Muscat Ottonel; $\mathrm{F}$ a. $=$ FeteascaAlba; $\mathrm{F} \mathrm{r}$. = Feteasca Regala; $\mathrm{F} \mathrm{n}$. $=$ Feteasca Neagra; $M=$ Merlot; $C$ s. = Cabernet Sauvignon.

As and Co content in wine ranged from $[21.85 \pm 1.74 \mu \mathrm{g} \mathrm{As} / \mathrm{L}$; $8.77 \pm 0.65 \mu \mathrm{g} \mathrm{As} / \mathrm{L}]$ and $[7.80 \pm 0.25 \mu \mathrm{g} \mathrm{Co} / \mathrm{L} ; 3.32 \pm 0.25 \mu \mathrm{g}$ Co $/ \mathrm{L}]$ with an average value of $11.87 \pm 1.37 \mu \mathrm{g} \mathrm{As} / \mathrm{L}$ and $5.17 \pm 0.51 \mu \mathrm{g} \mathrm{Co} / \mathrm{L}$. In both cases the maximum concentration were recorded in wine obtained from varieties cultivated in the Bujoru wine-growing center [21.85 $1.74 \mu \mathrm{g} \mathrm{As} / \mathrm{L}$ (Muscat Ottonel 2017); 7.80 $\pm 0.25 \mu \mathrm{g} \mathrm{Co} / \mathrm{L}$ (Cabernet Sauvignon 2015)] and in Smulti wine-growing center [21.12 $\pm 1.34 \mu \mathrm{g} / \mathrm{L}$ As (Feteasca Neagra 2017); $7.65 \pm 0.42 \mu \mathrm{g} / \mathrm{L}$ Co (Feteasca Alba 2016) and 7.23 $\pm 0.30 \mu \mathrm{g} / \mathrm{L}$ Co (Feteasca Neagra 2015)]. The average data shows that a higher concentration of As was obtained in white wines $(12.45 \mu \mathrm{g} / \mathrm{L})$ as compared to red wines $(11.42$ $\mu \mathrm{g} / \mathrm{L})$, while the higher concentration of Co was recorded for red wines $(5.58 \mu \mathrm{g} / \mathrm{L})$ as compared to white wines $(5.09 \mu \mathrm{g} / \mathrm{L})$ (Table 1). The results are comparable with those obtained in Serbia [13] $16.1 \mu \mathrm{g} / \mathrm{L}$ (As) for red wines and 9.46 (As) $\mu \mathrm{g} / \mathrm{L}$ for white wines, in Macedonia [14] $11.7 \mu \mathrm{g} / \mathrm{L}$ (As) by using the same microvinification technology, in Romania [15] $21.12 \mu \mathrm{g} / \mathrm{L}$ 
(As) and $154.90 \mu \mathrm{g} / \mathrm{L}(\mathrm{Sr})$, and higher compared to the results obtained in Italy [16] $2.91 \mu \mathrm{g} / \mathrm{L}$ for As, but for Sr the results obtained are significantly lower compared to the results obtained in Italy [16] $1340 \mu \mathrm{g} / \mathrm{L}(\mathrm{Sr})$. Regarding Co concentration the results agree with other studies made in Macedonia [12, 14] $3.9 \mu \mathrm{g} / \mathrm{L}$ and $13.90 \mu \mathrm{g} / \mathrm{L}$ for Co, and in Serbia [13] 3.89 $\mu \mathrm{g} / \mathrm{L}(\mathrm{Co})$ for red wines and 3.96 (Co) $\mu \mathrm{g} / \mathrm{L}$ for white wines.

The mean contents of $\mathrm{Ni}, \mathrm{Cr}$ and $\mathrm{Mn}$ were $312.32 \pm 2.42 \mu \mathrm{g} \mathrm{Ni} / \mathrm{L}$, $526.19 \pm 2.63 \mu \mathrm{g} \mathrm{Cr} / \mathrm{L}$ and $0.59 \pm 0.08 \mathrm{mg} \mathrm{Mn} / \mathrm{L}$, in case of $\mathrm{Ni}$ the highest concentrations were recorded in wine obtained from varieties cultivated in the Bujoru wine-growing centre Feteasca Alba (462.48 $\pm 4.02 \mu \mathrm{g} \mathrm{Ni} / \mathrm{L}$ (2017)) followed by the same variety but cultivated in the Oancea wine-growing center $(433.76 \pm 2.96 \mu \mathrm{g} \mathrm{Ni} / \mathrm{L}(2016))$. $\mathrm{Cr}$ was recorded the highest concentrations in wine obtained from varieties cultivated in the Oancea wine-growing center (923.62 $\pm 2.05 \mu \mathrm{g} \mathrm{Cr} / \mathrm{L}(2016)$ ), followed by the same variety but cultivated in the Bujoru wine-growing center (843.99 $\pm 3.89 \mu \mathrm{g} \mathrm{Cr} / \mathrm{L}$ (2016)). Regarding Mn concentration from wine samples, the highest concentrations in wine obtained from varieties cultivated in the Bujoru wine-growing center $(0.92 \pm 0.05 \mu \mathrm{g} \mathrm{Mn} / \mathrm{L}$ (2016)). The results agree with those made in Turkey [17] $520 \mu \mathrm{g} \mathrm{Ni} / \mathrm{L}$, in Romania [18] $147.73 \mu \mathrm{g} \mathrm{Cr} / \mathrm{L}$ and significantly higher than obtain in Serbia [8] $5.49 \mu \mathrm{g} \mathrm{Cr} / \mathrm{L}$ red wines and $6.56 \mu \mathrm{g} \mathrm{Cr} / \mathrm{L}$ white wines, in Italy [16] $54.57 \mu \mathrm{g} \mathrm{Ni} / \mathrm{L}$ and $19.68 \mu \mathrm{g} \mathrm{Cr} / \mathrm{L}$, in Macedonia [14] $32.10 \mu \mathrm{g} \mathrm{Cr} / \mathrm{L}$ and $10.20 \mu \mathrm{g} \mathrm{Cr} / \mathrm{L}$, in Romanian [18] 805.89 $\mu \mathrm{g} \mathrm{Mn/L.The} \mathrm{results} \mathrm{indicated} \mathrm{that} \mathrm{Romanian} \mathrm{wines} \mathrm{are}$ moderately rich in $\mathrm{Cr}$ (Table 2).

The concentration for $\mathrm{Cu}$ ranged from $0.45 \pm 0.10 \mathrm{mg} / \mathrm{L}$ to $0.90 \pm 0.04$ $\mathrm{mg} / \mathrm{L}$, with average values of $0.67 \pm 0.09 \mathrm{mg} / \mathrm{L}$, the latter value approaching the law limit $(1 \mathrm{mg} / \mathrm{L})$. The four samples [Merlot $0.82 \pm 0.07 \mathrm{mg} / \mathrm{L}$ Bujoru wine-growing center (2015), Feteasca Alba $0.83 \pm 0.05 \mathrm{mg} / \mathrm{L}$ Smulti winegrowing center (2015), Merlot $0.83 \pm 0.05 \mathrm{mg} / \mathrm{L}$ Smulti wine-growing center (2015) and Cabernet Sauvignon $0.83 \pm 0.08 \mathrm{mg} / \mathrm{L}$ Oancea wine-growing center (2015)] showed relatively high concentration of $\mathrm{Cu}$. Wide rages for $\mathrm{Cu}$ concentration in wines have been previously found and reported by other researchers in Brazil [19] 0.056-0.764 mg Cu/L, in Hungary [20] 0.031-0.313 $\mathrm{mg} \mathrm{Cu} / \mathrm{L}$, in Italy [21] $0.50-1.00 \mathrm{mg} \mathrm{Cu} / \mathrm{L}$ and in Greece [22] 0.076-0.114 mg Cu/L (Table 2).

Reporting the obtained results [Cd average $0.13 \pm 0.02 \mu \mathrm{g} / \mathrm{L}(0.1 \mathrm{mg} / \mathrm{kg}$ M.L.A. = Maximum Limit Allowed) Pb average $40.64 \pm 1.85 \mu \mathrm{g} / \mathrm{L}(0.15 \mathrm{mg} / \mathrm{kg}$ M.A.L); As average $11.87 \pm 1.37 \mu \mathrm{g} / \mathrm{L}(0.2 \mathrm{mg} / \mathrm{kg}$ M.A.L); Cu average $0.67 \pm 0.09 \mathrm{mg} / \mathrm{L}(1 \mathrm{mg} / \mathrm{kg} \mathrm{M} . \mathrm{A} . \mathrm{L})$ to national and international legislation we can say that the wine from Dealu Bujorului vineyard falls within the limits set by the law (Table 2). 
Table 2. Variation of the metal content of white and red wines from Dealu Bujorului Vineyard $(\mu \mathrm{g} / \mathrm{L})$

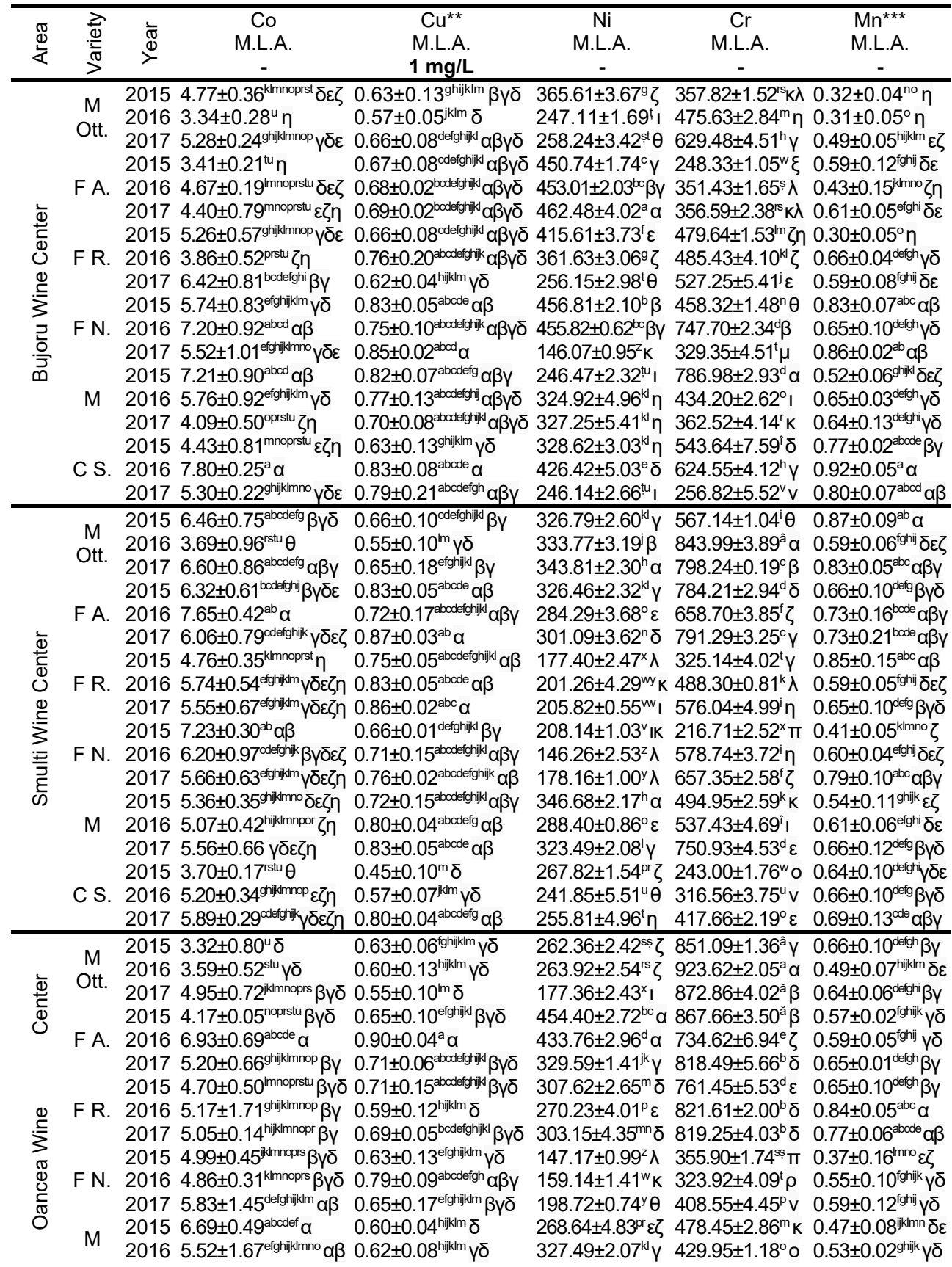




\begin{tabular}{|c|c|c|c|c|c|c|c|}
\hline 晃 & $\begin{array}{l}\frac{7}{0} \\
\frac{0}{\frac{1}{\pi}} \\
>\end{array}$ & 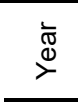 & $\begin{array}{l}\text { Co } \\
\text { M.L.A. } \\
\text { - }\end{array}$ & $\begin{array}{l}\mathrm{Cu}^{* *} \\
\text { M.L.A. } \\
1 \mathrm{mg} / \mathrm{L}\end{array}$ & $\begin{array}{c}\mathrm{Ni} \\
\text { M.L.A. } \\
-\end{array}$ & $\begin{array}{l}\text { Cr } \\
\text { M.L.A. } \\
-\end{array}$ & $\begin{array}{l}\text { Mn }{ }^{* \star \star} \\
\text { M.L.A. } \\
\quad-\end{array}$ \\
\hline & C S. & $\begin{array}{l}2017 \\
2015 \\
2016 \\
2017\end{array}$ & $\begin{array}{l}5.07 \pm 0.90^{\text {hijklmnopr }} \beta \gamma \\
4.16 \pm 0.20^{\text {noprstu }} \beta \gamma \delta \\
4.86 \pm 0.88^{\text {klmnoprs }} \beta \gamma \delta \\
5.47 \pm 1.15^{\text {fghijklmno }} \alpha \beta\end{array}$ & $\begin{array}{l}0.60 \pm 0.02^{\mathrm{hijklm}} \delta \\
0.83 \pm 0.08^{\mathrm{abcdef}} \mathrm{\alpha} \beta \\
0.59 \pm 0.12^{\mathrm{jkkm}} \delta \\
0.56 \pm 0.10^{\mathrm{klm}} \delta\end{array}$ & $\begin{array}{l}338.65 \pm 1.81^{i} \beta \\
264.48 \pm 1.53^{\mathrm{r}} \zeta \\
180.11 \pm 2.26^{\times} \theta \\
249.66 \pm 1.50^{\dagger} \eta\end{array}$ & $\begin{array}{l}520.36 \pm 1.50^{\mathrm{i}} \lambda \\
651.07 \pm 1.34^{\mathrm{f}} \eta \\
635.97 \pm 5.22^{\mathrm{g}} \theta \\
530.39 \pm 1.51^{\mathrm{j}}\end{array}$ & $\begin{array}{l}0.55 \pm 0.04^{\text {fghijk }} \gamma \delta \\
0.66 \pm 0.10^{\text {defgh }} \beta \gamma \\
0.27 \pm 0.03^{\circ} \zeta \\
0.34 \pm 0.03^{\text {mno }} \zeta\end{array}$ \\
\hline $\begin{array}{l}\text { A } \\
\text { Minir } \\
\text { Maxir }\end{array}$ & $\begin{array}{l}\text { vera } \\
\text { hum } \\
\text { hum }\end{array}$ & $\begin{array}{l}\text { ye } \\
\text { lalues } \\
\text { /alues }\end{array}$ & $\begin{array}{l}\mathbf{5 . 1 7} \pm \mathbf{0 . 5 1} \\
3.32 \pm 0.80 \\
7.80 \pm 0.25\end{array}$ & $\begin{array}{l}\mathbf{0 . 6 7} \pm \mathbf{0 . 0 9} \\
0.45 \pm 0.10 \\
0.90 \pm 0.04\end{array}$ & $\begin{array}{l}3 \mathbf{3 1 2 . 3 2} \pm \mathbf{2 . 4 2} \\
146.07 \pm 0.95 \\
462.48 \pm 4.02\end{array}$ & $\begin{array}{l}\mathbf{5 2 6 . 1 9} \pm \mathbf{2 . 6 3} \\
216.71 \pm 2.52 \\
923.62 \pm 2.05\end{array}$ & $\begin{array}{l}\mathbf{0 . 5 9} \pm \mathbf{0 . 0 8} \\
0.27 \pm 0.03 \\
0.92 \pm 0.03\end{array}$ \\
\hline & $\begin{array}{c}\mathrm{F} \\
\text { Sig. }\end{array}$ & & 7.227 & $\begin{array}{c}3.259 \\
* * *\end{array}$ & $\begin{array}{c}2799.869 \\
\star * \star\end{array}$ & $\underset{* \star \star}{9054.214}$ & 9.566 \\
\hline & & $\begin{array}{c}\mathrm{F} \\
\text { Sig. }\end{array}$ & $\underset{* * *}{11.926}$ & $\underset{* \star}{6.757}$ & $\underset{* * *}{12472.658}$ & $\underset{* * *}{36450.815}$ & $\underset{* * *}{19.617}$ \\
\hline Var & ety & $\begin{array}{c}\mathrm{F} \\
\text { Sig. }\end{array}$ & $\begin{array}{c}9.653 \\
* \star \star\end{array}$ & 7.235 & $\underset{* \star *}{8554.109}$ & $\underset{* \star *}{19135.062}$ & $\underset{* *}{3.668}$ \\
\hline & & $\begin{array}{c}\mathrm{F} \\
\text { Sig. }\end{array}$ & $\begin{array}{c}2.175 \\
\text { in }\end{array}$ & $\begin{array}{c}0.940 \\
\text { in }\end{array}$ & $\begin{array}{c}2630.180 \\
* * *\end{array}$ & $\begin{array}{c}3876.973 \\
* * *\end{array}$ & $\begin{array}{c}11.399 \\
\star * *\end{array}$ \\
\hline & $\begin{array}{l}{[18]} \\
{[23]} \\
{[15]} \\
{[24]}\end{array}$ & & $\begin{array}{c}2.08 \pm 1.19 \\
4.65 \\
2.50 \\
42.06\end{array}$ & $154.39 \pm 73.45^{\star \star}$ & $\begin{array}{c}18.39 \pm 1.19 \\
24.90\end{array}$ & $146.18 \pm 70.96$ & \\
\hline
\end{tabular}

Average value \pm standard deviation $(n=3)$. Romans letters represent the significance of the variety difference $(p \leq 0.05)$. Greeks letters represent the significance of the same variety cultivated in other year's difference ( $p$ $\leq 0.05)$. The difference between any two values, followed by at least one common letter, is insignificant. M.L.A. - maximum limit allowed (OIV, 2005); LOQ - lower than the limit of quantification; $\mathrm{Cu}^{*}=(\mathrm{mg} / \mathrm{L}) ; \mathrm{Cu}^{* *}=(\mu \mathrm{g} / \mathrm{L})$; $\mathrm{Mn}^{* * *}=(\mathrm{mg} / \mathrm{L}) . \mathrm{M}$ Ott. $=$ Muscat Ottonel; $\mathrm{F}$ a. $=$ FeteascaAlba; $\mathrm{F} \mathrm{r}$. = Feteasca Regala; $\mathrm{F} \mathrm{n}$. = Feteasca Neagra; $\mathrm{M}=$ Merlot; $\mathrm{C}$ s. $=$ Cabernet Sauvignon.

\section{Combining multielement analysis from wine samples for discrimination analysis}

Multivariate chemometric method was used as a supervised learning technique for the differentiation of wines intro groups on the basis of grape variety and year of production and finding markers which showed a significant discrimination value (variables with Wilk's lambda near zero, $p$ value $<0.05$ and higher $F$ coefficients). Stepwise linear discriminant analysis (LDA) was used to identify significant tracers for classification to the geographical discrimination of the wines samples. Stepwise Discriminant Analysis (LDA) was used to designate suitable variables for classification of the samples, eliminating the variables that do not contribute to discrimination of the wine.

In order to validate the proposed statistic model, based on variables which showed higher significance in first LDA assessment, we performed a second Liniar Discriminant Analysis (LDA) for the test set consisting of wines used to build statistical model (training set) together with data from other wine samples that are not included in the first LDA (control-set). Cross-validation was applied to determine the optimal number of variables required to obtain robust models. 


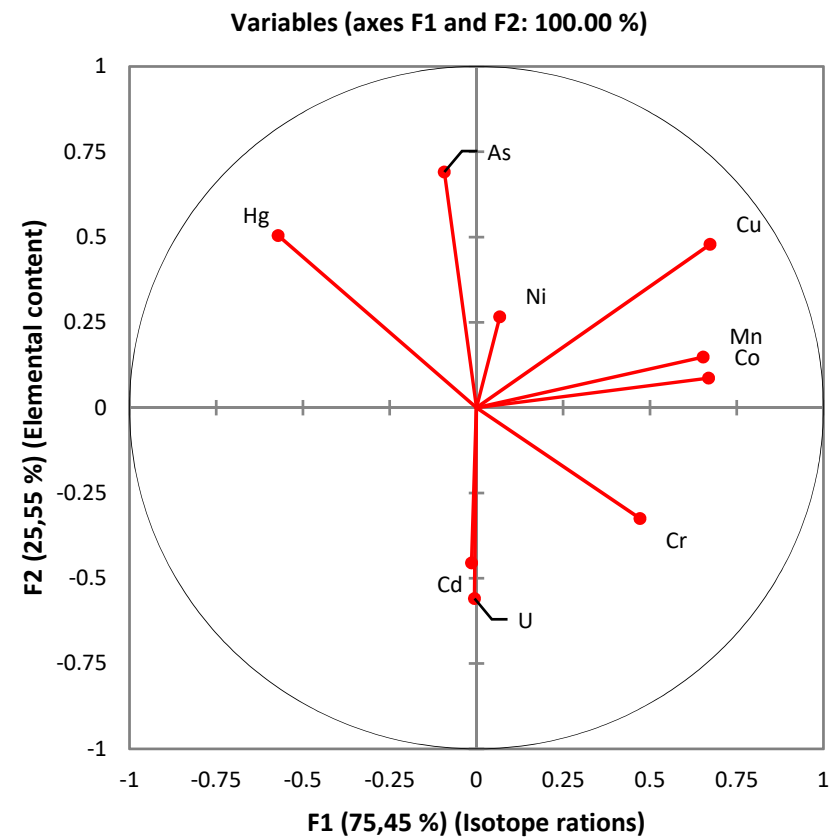

Figure 1. Correlation between analyzed parameters and the factors in discriminant analysis the origin of the wine

In this study, the content of certain wines shows high concentration of metals, but not exceeding the maximum recommended by International Organisation of Vine and Wine [25], and this mostly due to agricultural practices, fertilizers and technological winemaking processes.

Elements like $\mathrm{Pb}, \mathrm{Cu}, \mathrm{Ni}, \mathrm{Cd}, \mathrm{U}$ and $\mathrm{Hg}$ showed a high discriminatory power for geographic origin of Romanian wines, but additional new elements $(\mathrm{Mn}, \mathrm{Cr}$ ) have been investigate in order to identify new tracers for geographical traceability of Romanian wines. The wines obtained in the three wine-growing centers can be geographical fingerprints based on the concentration of $\mathrm{Pb}, \mathrm{Cu}, \mathrm{Ni}, \mathrm{Cd}, \mathrm{Hg}, \mathrm{Mn}, \mathrm{Cr}, \mathrm{U}$.

The cross-validation technique provided a $100 \%$ percentage of predicted membership according to the origin of the wine (F1 $=75.45 \%$ and F2 $=25.55 \%$ ). The linear correction revealed acceptable scores for the two defined discriminant factors (F1 and F2) (Figure 1). A significant differentiation of wines according to wine-growing centers and year of wine production was carried out for wines samples, which demonstrates the importance of elemental profile for the geographical traceability of wines (Figure 2). 


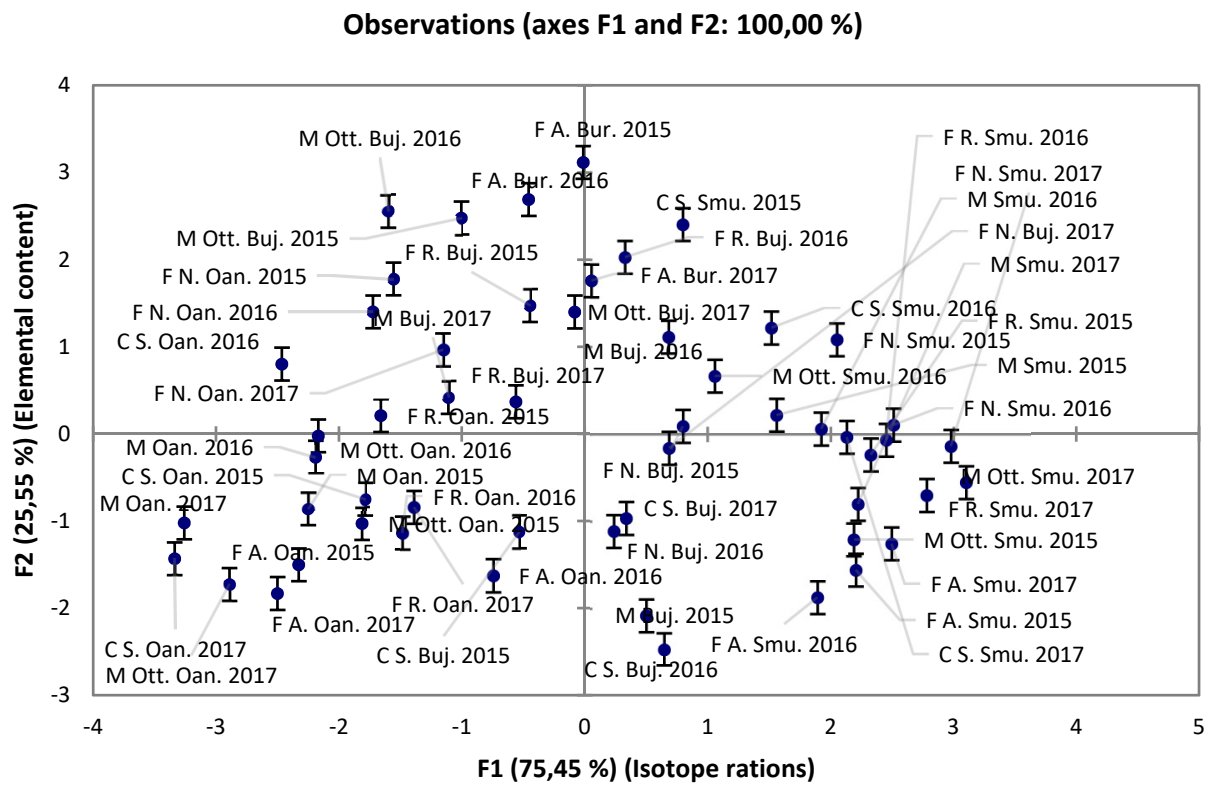

Figure 2. Differentiation of wines based on element contents

Heat map was used to discover sample groups, discover groups and also to discover related sample/feature groups. In case of elemental contents from wine, the dendrogram (horizontal dendrogram) clearly show two cluster, first cluster is formed from the Co, Cd, U, Hg, Mn, Cu, As and second cluster was formed from $\mathrm{Ni}$ an $\mathrm{Cr}$. Based on this distribution it can be seen that the $\mathrm{Cr}$ recorded the highest concentration followed by $\mathrm{Ni}$. The vertical dendrogram show also two cluster, the first M Smu. 2015, C S. Buj. 2015, F R. Buj. 2015, M Buj. 2017, M Oan. 2016, M Oan. 2015, F R. Smu. 2015, F R. Buj. 2017, C S. Smu. 2017, F A. Buj. 2017, F A. Buj. 2015, C S. Buj. 2017, C S. Smu. 2016, F N. Oan. 2016, F N. Oan. 2015 and second cluster was formed from F A. Smu. 2017, M Ott. Smu. 2017, M Ott. Smu. 2016, F R. Oan. 2015, M Ott. Oan. 2017, F R. Oan. 2016, M Buj. 2015, F A. Oan. 2016, F R. Smu. 2017, C S. Oan. 2016, F A. Smu. 2016, M Ott. Buj. 2017. Based on this distribution in can be seen that there is a separation of wine varieties depending on elemental contents, except for a few varieties that do not fit into this rule (F R. Buj. 2015, F R. Smu. 2016, F R. Buj. 2017, F A. Buj. 2017, F A. Buj. 2015 (which have been introduced in red wine cluster) and M Buj. 2015, C S. Oan. 20162015 (which have been introduced in white wine cluster) (Figure 3). 


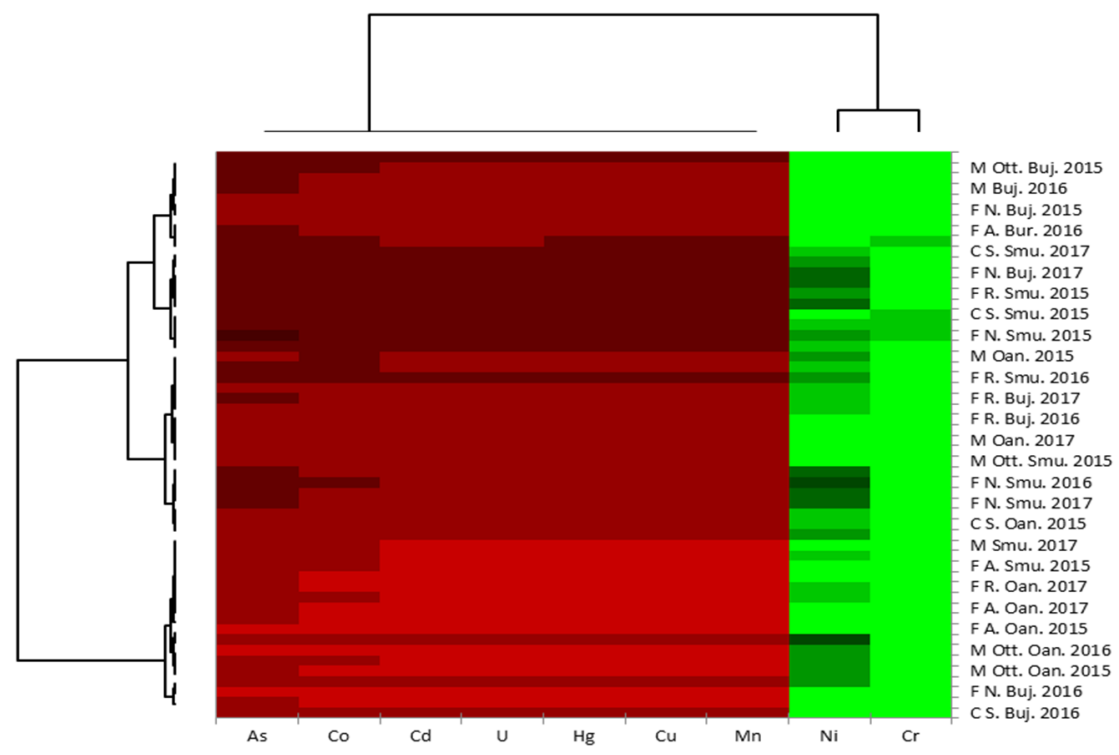

Figure 3. Heat map obtained by cluster analysis of the element contents

\section{CONCLUSIONS}

In this work the elemental composition of white wines (Muscat Ottonel, Feteasca Alba, Feteasca Regala) and red wines (Feteasca Neagra, Merlot, Cabernet Sauvignon) production years 2014-2016 from Bujoru, Smulti and Oancea wine-growing centers was studied in order to highlight geographical traceability of elemental composition for fingerprints of the wines.

Concentration of $\mathrm{Cd}, \mathrm{Pb}, \mathrm{As}$ and $\mathrm{Cu}$ heavy metals in analysed wine samples were under Maximum Limit Allowed (M.L.A.), respectively as published by the Organization of Vine and Wine. The content of potentially toxic elements such as $\mathrm{Cd}, \mathrm{Pb}, \mathrm{U}, \mathrm{Hg}, \mathrm{As}, \mathrm{Cu}, \mathrm{Ni}, \mathrm{Cr}$ and $\mathrm{Mn}$ are lower than the recommended values found in literature, highlighting the safety and quality of the analysed Romanian wines.

\section{EXPERIMENTAL SECTION}

\section{Study area}

A total of 162 wine samples were analysed ( 3 white wines and 3 red wines). Samples originated from Bujoru, Smulti and Oancea wine-growing centers part of Dealu Bujorului vineyard ( $\left.45^{\circ} 52^{\prime} 10^{\prime \prime} \mathrm{N}, 27^{\circ} 55^{\prime} 8^{\prime \prime} \mathrm{E}\right)$. The Dealu 
Bujorului vineyard is characterized by an alternate landscape, from flat to hilly areas, with altitude between 100 and $225 \mathrm{~m}$ and the predominant soil is levigated chernozem having a clayey sand texture with $\mathrm{pH}$ between values 7.4 and 8.1. Although they have moisture deficit, natural conditions (ecoclimatic and ecopedological) offer viable ecosystem for the development of vineyard. The vineyard is crossed by the parallel $46^{\circ}$ latitude north, intersected by the $28^{\circ}$ longitude meridian. Dealu Bujorului vineyard belongs to Galați country. The specificity of the transition area is highlighted by the predominance of deposits of clays and sands. Versants were made from clay deposits and sandy sands.

\section{Sample collection and microvinification process}

The samples used in this experiment were obtained from the wines produced from Muscat Ottonel, Feteasca Alba, Feteasca Regala, Feteasca Neagra, Merlot and Cabernet Sauvignon under the conditions of 2015, 2016 and 2017. The wine samples resulted from micro-wine production. Micro-wine production was done according to the methodology describe by Bora et al. [26]. All wines were providing by the wineries as finished wines in $750 \mathrm{~mL}$ bottles with cork stoppers and were stored at $3-4^{\circ} \mathrm{C}$ before analysis. All vines were planted since 1979, and the vine plantation was organized with $2.2 \times 1 \mathrm{~m}$ distance between rows and plants. Vines were pruned according to the Guyot system and were grown on speliers.

\section{Reagents and solutions}

Ten elements ( $\mathrm{Cd}, \mathrm{Pb}, \mathrm{U}, \mathrm{Hg}, \mathrm{As}, \mathrm{Sr}, \mathrm{Co}, \mathrm{Cu}, \mathrm{Ni}, \mathrm{Cr}$ and $\mathrm{Mn}$ ) were determined in order to assess their ability to discriminate wines by geographical origin. The analysis was made using multielement analysis and ICP-MS technique, after an appropriate dilution, using external standard calibration method. The calibration was performed using XXICertiPUR multielement standard, and from individual standard solution of $\mathrm{Cr}$ and $\mathrm{Hg}$. The working standards and the control sample were prepared daily from the intermediate standards that were prepared from the stock solution. The intermediate solutions stored in polyethylene bottles and glassware was cleaned by soaking in $10 \% \mathrm{v} / \mathrm{v} \mathrm{HNO}_{3}$ for 24 hours and rinsing at least ten times with ultrapure water $\left(18.2{\mathrm{M} \Omega \mathrm{cm}^{-1}}^{-}\right.$ultrapure water-Types 1$)$. The accuracy of the methods was evaluated by replicate analyses of fortified samples $(10 \mu \mathrm{L}-10 \mathrm{~mL}$ concentrations) and the obtained values ranged between 0.8-13.1 percent, depending on the element. The global recovery for each element was estimated and the obtained values were between 84.6-100.9\% [27]. 
For quality control purpose, blanks and triplicates samples $(n=3)$ were analyzed during the procedure. The variation coefficient was under $5 \%$ and detection limits ( $p p b)$ were determined by the calibration curve method. Limit of detection (LoD) and Limit of quantification (LoQ) limits were calculated according to the next mathematical formulas: $L o D=3 S D / s$ and $\mathrm{LoQ}=10 \mathrm{SD} / \mathrm{s}$ (SD = estimation of the standard deviation of the regression line; $s=$ slope of the calibration curve) $($ Table 3 ).

Table 3. Instrumental conditions for the determination of each element (ICP-MS technique)

\begin{tabular}{|c|c|c|c|c|}
\hline Element & $\begin{array}{c}\text { Correlation } \\
\text { coefficient }\end{array}$ & $\begin{array}{c}\mathbf{L o D}^{*} \\
(\boldsymbol{\mu g} / \mathbf{L})\end{array}$ & $\begin{array}{c}\text { LoQ } \\
(\boldsymbol{\mu g} / \mathbf{L})\end{array}$ & $\begin{array}{c}\mathbf{B E C}^{* * *} \\
(\boldsymbol{\mu g} / \mathbf{L})\end{array}$ \\
\hline $\mathrm{Cd}$ & 0.9999 & 0.0202 & 0.0673 & 0.027 \\
\hline $\mathrm{U}$ & 0.9999 & 0.0253 & 0.0842 & 0.005 \\
\hline $\mathrm{As}$ & 0.9999 & 0.2335 & 0.7776 & 0.538 \\
\hline $\mathrm{Co}$ & 0.9999 & 0.0365 & 0.1215 & 0.152 \\
\hline $\mathrm{Ni}$ & 0.9999 & 0.0591 & 0.1968 & 0.091 \\
\hline $\mathrm{Pb}$ & 0.9999 & 0.0003 & 0.0010 & 0.002 \\
\hline $\mathrm{Hg}$ & 0.9999 & 0.0417 & 0.1379 & 0.128 \\
\hline $\mathrm{Sr}$ & 0.9999 & 0.1434 & 0.4775 & 0.955 \\
\hline $\mathrm{Cu}$ & 0.9999 & 0.0402 & 0.1339 & 0.237 \\
\hline $\mathrm{Cr}$ & 0.9999 & 1.6630 & 5.5378 & 0.636 \\
\hline $\mathrm{Mn}$ & 0.9999 & 0.0100 & 0.0340 & 0.085 \\
\hline
\end{tabular}

*Detection limit; ${ }^{* *}$ Background equivalent concentration; ${ }^{* * *}$ Quantification limit.

\section{Sample preparation for determination of heavy metals and isotopic ration from wine using ICP-MS.}

For the determination of elements from wine samples were used an amount of $0.5 \mathrm{~mL}$ wine and diluted to $8 \mathrm{~mL}\left(7 \mathrm{~mL} \mathrm{HNO} 365 \%+1 \mathrm{~mL} \mathrm{H}_{2} \mathrm{O}_{2}\right.$ ) the obtained solutions were placed in a clean Teflon digestion vessel, after 15-30 minutes the mineralization was performed using a microwave system Milestone START D Microwave Digestion System set in three steps: step I (time $10 \mathrm{~min}$., temperature $200^{\circ} \mathrm{C}$ ), step II (time $15 \mathrm{~min}$., temperature $200^{\circ} \mathrm{C}$ ) and step III (time $40 \mathrm{~min}$., ventilation - temperature $32^{\circ} \mathrm{C}$ ). After mineralization, samples were filtered through a $0.45 \mathrm{~mm}$ filter and brought to a volume of $50 \mathrm{~mL}$ with $\mathrm{HNO}_{3} 1 \%$.

In order to confirm the best-chosen conditions for wine digestion standard additions for checking accuracy of the microwave digestion and recoveries were calculated (Table 4). The digestion seemed visually completed in all of the combinations, but the spiked recoveries showed significant differences for total elements content $(p-$ Value $=0.005)$. 
Table 4. Standard additions for checking accuracy of the microwave digestion ICP-MS method $(n=3)$ (SRM 1643e)

\begin{tabular}{|c|c|c|}
\hline Element & $\begin{array}{c}\text { Certified Concentration } \\
(\mathrm{mg} / \mathrm{L})\end{array}$ & $\begin{array}{c}\text { Measured Concentration } \\
(\mathrm{mg} / \mathrm{L})\end{array}$ \\
\hline $\mathrm{Cd}$ & $6.568 \pm 0.073$ & $6.473 \pm 0.106$ \\
\hline $\mathrm{U}$ & $9.994 \pm 0.016$ & $9.981 \pm 0.012$ \\
\hline $\mathrm{As}$ & $56.85 \pm 0.37$ & $53.09 \pm 0.31$ \\
\hline $\mathrm{Co}$ & $27.06 \pm 0.28$ & $24.13 \pm 0.06$ \\
\hline $\mathrm{Ni}$ & $62.41 \pm 0.69$ & $61.32 \pm 0.21$ \\
\hline $\mathrm{Pb}$ & $19.63 \pm 0.21$ & $19.13 \pm 0.09$ \\
\hline $\mathrm{Hg}$ & $0.1016 \pm 0.0017$ & $0.1102 \pm 0.0012$ \\
\hline $\mathrm{Sr}$ & $314.00 \pm 19.00$ & $314.09 \pm 09.06$ \\
\hline $\mathrm{Cu}$ & $21.44 \pm 0.70$ & $21.25 \pm 0.21$ \\
\hline $\mathrm{Cr}$ & $18.32 \pm 0.10$ & $19.18 \pm 0.21$ \\
\hline $\mathrm{Mn}$ & $38.02 \pm 0.44$ & $33.04 \pm 0.05$ \\
\hline
\end{tabular}

\section{Instrumentation}

The determination of metals was performed on mass spectrometer with inductively coupled plasma, (ICP-MS) iCAP Q Thermo scientific model, based polyatomic species before they reach the quadrupole mass spectrometer, using a PFA micro flow concentric nebulizer. The argon used was of $99.99 \%$ purity (Messer, Austria). The instrument was daily optimized to give maximum sensitivity for $\mathrm{M}^{+}$ions and the double ionization and oxides monitored by the means of the rations between $\mathrm{Ba}^{2+} / \mathrm{Ba}^{+}$and $\mathrm{Ce}^{2+} / \mathrm{CeO}^{+}$, respectively, these always being less than $2 \%$. The experimental conditions were: argon flow on nebulizer $(0.82 \mathrm{~L} / \mathrm{min}$.), auxiliary gas flow $0.80 \mathrm{~L} / \mathrm{min}$., argon flow in plasma $15 \mathrm{~L} / \mathrm{min}$., lens voltage $7.30 \mathrm{~V}$; RF power in plasma 1100 $\mathrm{W}$, spray chamber temperature $\left(2.42 \pm 1.00^{\circ} \mathrm{C}\right)$. Accuracy was calculated for the elements taken into consideration (0.5-5.0\%).

\section{Statistical analysis}

The statistical interpretation of the results was performed using the Duncan test, SPSS Version 24 (SPSS Inc., Chicago, IL., USA). The statistical processing of the results was primarily performed in order to calculate the following statistical parameters: average and standard deviation. This data was interpreted with the analysis of variance (ANOVA) and the average separation was performed with the DUNCAN test at $p \leq 0.05$. Multivariate chemometric method was used as a supervised learning technique for the differentiation of wines intro groups on the basis of grape variety and year 
of production and finding markers which showed a significant discrimination value (variables with Wilk's lambda near zero, $p$ value $<0.05$ and higher $F$ coefficients). Stepwise linear discriminant analysis (LDA) was used to identify significant tracers for classification to the geographical discrimination of the wines samples. Stepwise Discriminant Analysis (LDA) was used to designate suitable variables for classification of the samples, eliminating the variables that do not contribute to discrimination of the wine. In order to validate the proposed statistic model, based on variables which showed higher significance in first LDA assessment, we performed a second Liniar Discriminant Analysis (LDA) for the test set consisting of wines used to build statistical model (training set) together with data from other wine samples that are not included in the first LDA (control-set). Cross-validation was applied to determine the optimal number of variables required to obtain robust models. Linear discriminant analysis (LDA) was performed using Microsoft Excel 2016 and XLSTAT Addinsoft version 15.5.03.3707.

\section{REFERENCES}

1. D. M. A. M. Luykx, S. M. Van Rush, Food Chemistry, 2008, 107, 897.

2. C. M. R. Almeida, M. T. S. D. Vasconcelors, Journal of Agricultural and Food Chemistry, 2003, 51, 4788.

3. M. Álvarez, J. M. Moreno, A. M. Jos, A. M. Cameán, G. González, Journal of Food Composition and Analysis, 2007, 20, 391.

4. R. D. Di Paola-Naranjo, M. V. Baroni, N. S. Podio, H. R. Rubinstein, M. P. Fabani, R. G. Badini, M. Inga, H. A. Ostera, M. Cagnoni, E. Gallegos, E. Gautier, P. Peral-Garcia, J. Hoogewerff, D. A. Wunderlin, Journal of Agricultural and Food Chemistry, 2011, 59(14), 7854.

5. I. S. Arvanitoyannis, M. N. Katsota, E. P. Psarra, E. H. Souflerous, S. Kallithraka, Trends in Food Science and Technology, 1999, 10, 321.

6. J. D. Greenough, H. P. Longerich, S. E. Jackson, Australian Journal of Grape and Wine Research, 1997, 3, 75.

7. M. M. Castiñeira-Gomez, R. Brant, N. Jakubowski, J. T. Andersson, Journal of Agricultural and Food Chemistry, 2004, 52, 2953.

8. M. Fabani, M. Toro, F. Vazquez, M. P. Díaz, D. A. Wunderlin, Journal of Agricultural and Food Chemistry, 2009, 57, 7409.

9. F. Galgano, F. Favati, M. Caruso, T. Scarpa, A. Palma, LWT-Food Science and Technology, 2008, 41, 1808.

10. P. Kment, M. Mihaljevic, V. Ettler, O. Šebek, L. Strnad, L. Rohlavá, Food Chemistry, 2005, 91, 157.

11. R. Larcher, G. Nicolini, P. Pangrazzi, Journal of Agricultural and Food Chemistry, 2003, 51(20), 5956. 
12. V. Ivanova-Petropulos, H. Wiltsche, T. Stafilov, M. Stefova, H. Motter, E. Lankmayr, Macedonian Journal of Chemistry and Chemical Engineering, 2013, 32(2), 265.

13. S. Đurđic, M. Pantelic, J. Trifkovic, V. Vukojevic, M. Natic, 'Z. Tesica, J. Mutic, RSC Advanced, 2017, 7, 2151.

14. V. Ivanova-Petropulos, B. Balavanova, S. Mitrev, D. Nedelkovski, V. Dimovska, R. Gulaboski, Food Analytical Methods, 2016, 9(1), 48.

15. V. Avram, C. Voica, A. Hosu, C. Cimpoiu, C. Măruțoiu, Revue Roumaine de Chimie, 2014, 59(11-12), 1009.

16. F. Galgano, F. Favati, M. Caruso, T. Scarpa, A. Palma, LWT-Food Science and Technology, 2008, 41, 1808.

17. D. Karataș, F. Aydin, I. Aydin, H. Karataș, Czech Journal of Food Sciences, 2015, 33(3), 228.

18. E. I. Geana, A. Marinescu, A. M. Iordache, C. Sandru, R. E. Ionete, C. Bala, Food Analytical Methods, 2014, 48(2), 2064.

19. D. Schiavo, I.Y. Neira, I.A. Nóbrega, Talanta, 2008, 76, 1113.

20. Z. Ajtony, N. Szoboszlai, E.K. Suskó, P. Mezei, K. György, L. Bencs, Talanta, 2008, 76, 627.

21. G. Dugo, L. La Pera, T.M Pellicanó, G. Di Bella, M. D'Imperio, Food Chemistry, $2005,91,355$.

22. S. Galani-Nikolakaki, N. Kallithrakas-Kontos, A.A. Katsanos, Science of the Total Environment, 2002, 285, 155.

23. G. Thiel, G. Geisler, I. Blechschmidt, K. Danzer, Analytical and Bioanalytical Chemistry, 2004, 378, 1630.

24. I. Geana, A. Iordache, R. Ionete, A. Marinescu, A. Ranca, M. Culea, Food Chemistry, 2013, 138, 1125.

25. OIV, International Code of Oenological Practices, Annex: Maximum Acceptable Limits, 2015, Issue 2015/01. Paris, France.

26. F. D. Bora, A. Donici, T. Rusu, A. Bunea, D. Popescu, C. I. Bunea, Notulae Botanicae Horti Agrobotanici Cluj-Napoca, 2018, 46(1), 223.

27. E.I. Geana, C. Sandru, V. Stanciu, R.E. Ionete, Food Analytical Methods, 2017, 10(1), 63. 